\title{
Morphological Changes of Rat Jejunum after Whole Body $\gamma$-irradiation and Their Impact in Biodosimetry
}

\author{
D. DRIÁK ${ }^{1,2}$, J. ÖSTERREICHER ${ }^{2}$, J. VÁVROVÁ ${ }^{2}$, Z. ŘEHÁKOVÁ ${ }^{2}$, Z. VILASOVÁ ${ }^{2}$ \\ ${ }^{1}$ Clinic of Gynaecology and Obstetrics, First Faculty of Medicine, Charles University and \\ University Hospital Bulovka, Prague, ${ }^{2}$ Department of Radiobiology and Molecular Pathology, \\ Faculty of Military Health Sciences, University of Defense, Hradec Králové, Czech Republic
}

Received May 16, 2006

Accepted March 8, 2007

On-line April 25, 2007

\section{Summary}

Gastrointestinal form is the second stage of the Acute Radiation Syndrome (ARS) with a threshold dose of $8 \mathrm{~Gy}$. It represents an absolutely lethal clinical-pathological unit, enteritis necrohemorrhagica (duodenitis, jejunitis, ileitis, respectively) with unknown causal therapy. The purpose of our study has been to evaluate the morphological changes in a model of radiationinduced enteritis in rats and estimate the significance of changes in biodosimetry. Wistar rats were randomly divided into 21 groups, 10 animals per group. Samples of the jejunum were taken $24,48,72$, and $96 \mathrm{~h}$ after the whole-body $\mathrm{y}$-irradiation with the doses of $1,5,10,15$, and $20 \mathrm{~Gy}$, and routinely stained with hematoxylin and eosin. Five morphometric markers intercryptal distance, enterocytal height on the top and base of villus, length of basal lamina of 10 enterocytes and enterocytal width - in irradiated rat jejunum were examined. The results were compared with sham-irradiated control group. After lethal doses of irradiation, all morphometric parameters of jejunum significantly changed. With the exception of intercryptal distance, they might be considered as suitable biodosimetric markers under these experimental conditions. Our morphometry results in radiation-induced jejunitis are in accordance with those in other studies. We were the first who quantified morphological postirradiation changes in animal jejunum. Some of them might be used under experimental conditions. This experimental study is a predecessor of the clinical assessment of a specific marker. Under clinical practice, the sensitive biodosimetric parameter could serve as one of the guidance for evaluation of the absorbed dose in irradiated troops as well as rescue workers. This is in accordance with tasks and Standardization Agreement of the North Atlantic Treaty Organization.

\section{Key words}

Radiation-induced enteritis - Threshold dose • Biodosimetric marker

\section{Corresponding author}

Daniel Driák, Gynaecological-Cbstetric Clinic, University Hospital Bulovka, Budínova 2, 180 81, Praha 8, Czech Republic. Fax: +420 28384 0507. E-mail: driak@seznam.cz

\section{Introduction}

Large devastating effects of ionizing radiation on cell renewal systems, e.g. intestinal epithelium, have been known for more than 100 years (Regaud et al. 1912, Walsh 1897). Radiation-induced enteritis is an absolutely lethal clinical-pathological syndrome whose effective causal therapy remains unknown. Target radiosensitive cells are represented by hitherto immunohistochemically undefined enterocytal stem cells localized on the bottom of the crypts of Lieberkühn. Ascending from this location the cells lose the proliferative property and mature into differentiated enterocytes, which cover the top of the villus (Classen et al. 1998). Under physiological conditions, the matured enterocytes are successively eliminated into the intestinal lumen and replaced by the new cells from the crypt. After 5-8 days of irradiation with a dose of 8 Gy and higher, the inflammatory cascade, denudation of epithelium, and other morphological changes of the intestinal surface develop (Figs 1 and 2). The damaged intestinal epithelium loses its function and septic shock becomes the most common cause of death (Brennan et al. 1998, Buell and Harding 1989, Fajardo 1982, MacNaughton 2000).

However, the quantification of radiation-induced enteropathy has not been described yet. The objective of our study was to evaluate, by a standard procedure of radiation-induced enteritis, the morphological changes in 


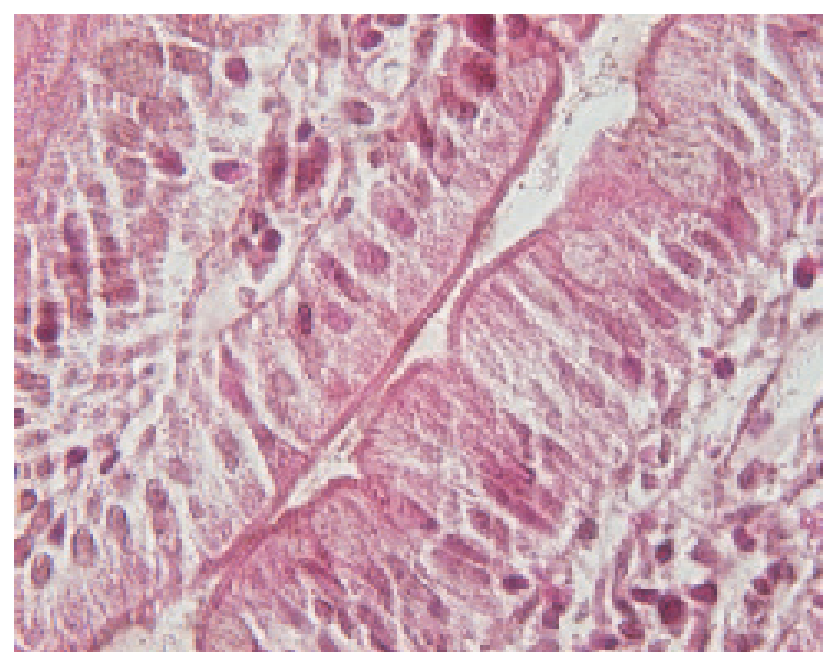

Fig. 1. Sham-irradiated jejunum, 800 -fold, hematoxylin-eosin

rat jejunum after whole-body $\gamma$-irradiation with the doses of $1,5,10,15$, and 20 Gy at $24,48,72$, and $96 \mathrm{~h}$ after the exposure, respectively. Furthermore, we wanted to estimate the significance of morphological changes in biodosimetry.

We have chosen the jejunal epithelium because its anatomy and physiology enables more distinct cell kinetics and minute description of enterocytes than other parts of the gastrointestinal tract. In addition, it represents a very dynamic part of the gastrointestinal tract with active enzymatic secretion. Consequently, the changes of enzymatic secretion depending on ionizing radiation will be examined, which is impossible in other parts of gastrointestinal tract.

\section{Methods}

A total of 210 adult male Wistar rats aged 12-16 weeks weighing $250-300 \mathrm{~g}$ were randomly allocated to 21 groups. Under anesthesia with thiopental, the animals were irradiated with whole body $\gamma$-rays of ${ }^{60} \mathrm{Co}$ unit (Chisotron Chirana) in a dose rate of $1.0 \mathrm{~Gy} / \mathrm{min}$ at a distance of $100 \mathrm{~cm}$ from the skin with the doses of 1,5 , 10, 15, and $20 \mathrm{~Gy}$. The animals were killed by cervical dislocation under ether anesthesia 24, 48, 72, and $96 \mathrm{~h}$ after irradiation. A sham-irradiated control group was sacrificed after $72 \mathrm{~h}$. All the procedures involving animals were approved by the Ethics Committee of the Faculty of Military Health Sciences in Hradec Králové.

\section{Histological examination}

Samples of proximal jejunum (approximately $2 \mathrm{~cm}$ aborally from duodeno-jejunal flexure) were

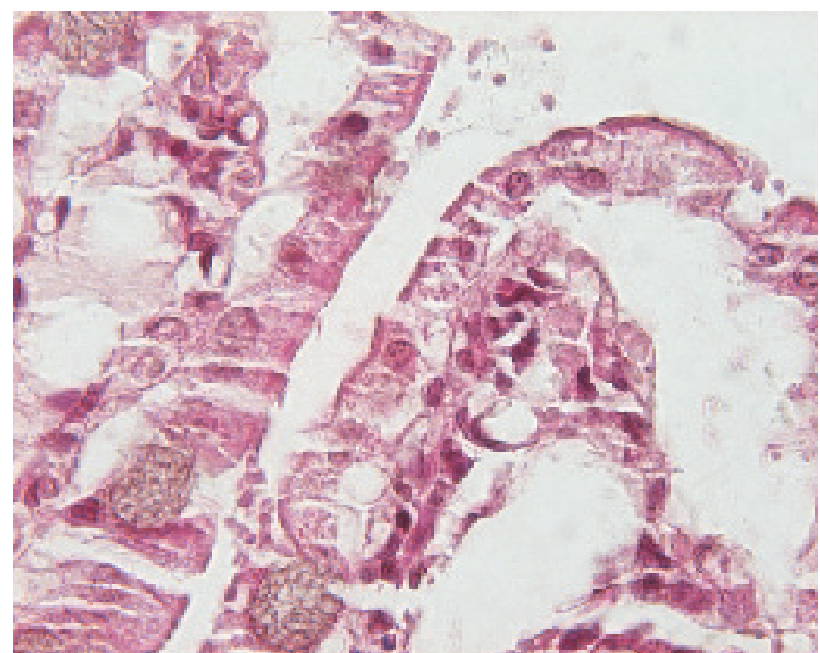

Fig. 2. Jejunum $72 \mathrm{~h}$ after irradiation with the dose of $20 \mathrm{~Gy}$, 800-fold, hematoxylin-eosin

extirpated and routinely processed for microscopical examination. The samples were fixed with $10 \%$ neutral buffered formalin up to $24 \mathrm{~h}$ and embedded into paraffin. One $\mu \mathrm{m}$ thick tissue sections of jejunum were stained with hematoxylin and eosin. Samples from each animal were stained with Gram's staining for excluding bacterial invasion.

\section{Measurement of morphometric parameters}

Stained samples were evaluated using a BX-51 microscope (Olympus Company, Prague, Czech Republic). Under the microscope, the intercryptal distance (magnified 200-fold), height of enterocyte on the top and at the base of villi, length of basal lamina of ten enterocytes and width of enterocytes (magnified 800fold) were measured. Ten measurements were performed per one animal, so we had 100 measurements in each group. All values (mean $\pm 2 \times$ S.E.M.) were calculated in micrometers $(\mu \mathrm{m})$.

\section{Data processing}

The Mann-Whitney test was used for the statistical analysis, giving the mean $\pm 2 \times$ S.E.M., that express $95 \%$ confidence interval.

\section{Results}

Intercryptal distance in irradiated jejunum (Fig. 3)

In comparison with non-irradiated animals, the values of intercryptal distance in rats $72 \mathrm{~h}$ after irradiation with the dose of $10 \mathrm{~Gy}$ and in all animals irradiated with the doses of 15 and 20 Gy were significantly higher with 


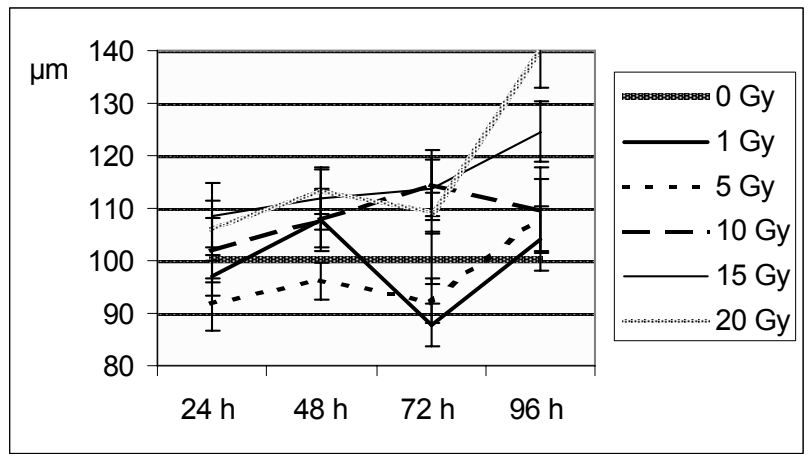

Fig. 3. Intercryptal distance in jejunum.

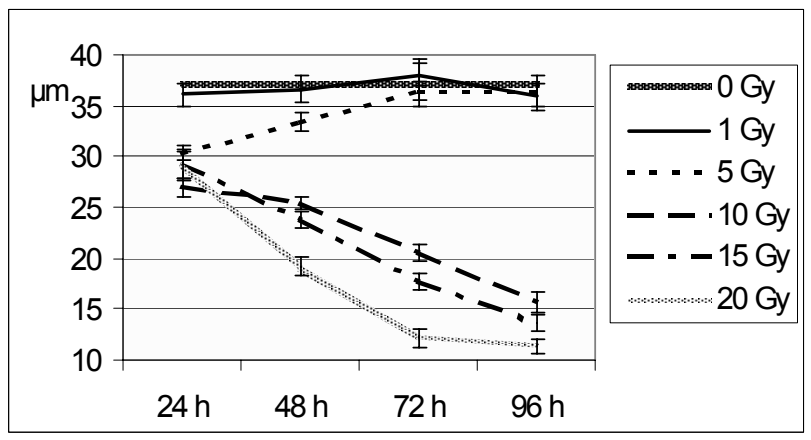

Fig. 4. Height of enterocyte on the top of villus.

maximum $96 \mathrm{~h}$ after irradiation with the dose of $20 \mathrm{~Gy}$. A significantly lower value was found in rats irradiated with the dose of $1 \mathrm{~Gy}$ after $72 \mathrm{~h}$.

\section{Enterocyte height on the top of villi in jejunum (Fig. 4)}

In comparison with non-irradiated animals, the values of enterocyte height were significantly lower in rats examined 24-48 $\mathrm{h}$ after irradiation with the dose of $5 \mathrm{~Gy}$ and in all time intervals in all animals irradiated with the dose of 10 Gy and higher with maximum decrease observed at the time interval of $96 \mathrm{~h}$ after irradiation with the dose of $20 \mathrm{~Gy}$. The dose-related diminishing of enterocyte height was observed 48-96 h after irradiation with the doses of 5-20 Gy.

\section{Enterocyte height on the base of villi in jejunum (Fig. 5)}

In comparison with non-irradiated animals, the values of enterocyte height were significantly lower in rats examined 24-48 $\mathrm{h}$ after irradiation with the dose of $1 \mathrm{~Gy}, 24 \mathrm{~h}$ after irradiation with the dose of $5 \mathrm{~Gy}$, at all time intervals after irradiation with the dose of $10 \mathrm{~Gy}$ and higher, and 48-96 $\mathrm{h}$ after irradiation with the dose of 15 Gy. Maximum decrease was observed at the time interval of $96 \mathrm{~h}$ after irradiation with the dose $20 \mathrm{~Gy}$. From the dose of 15 Gy and higher, the time-related

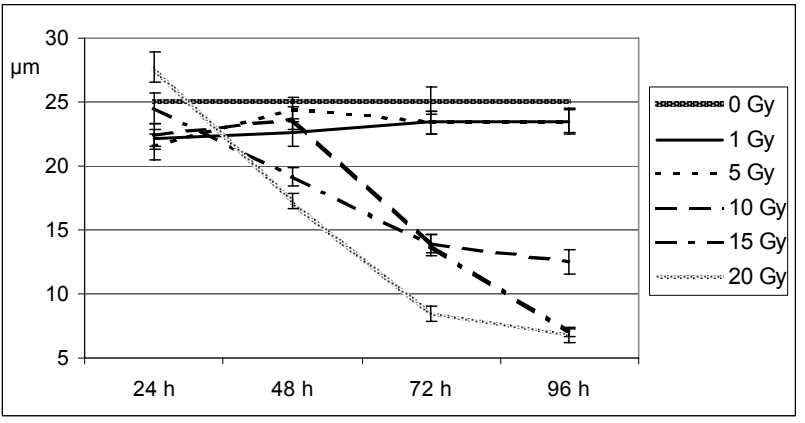

Fig. 5. Height of enterocyte on the base of villus.

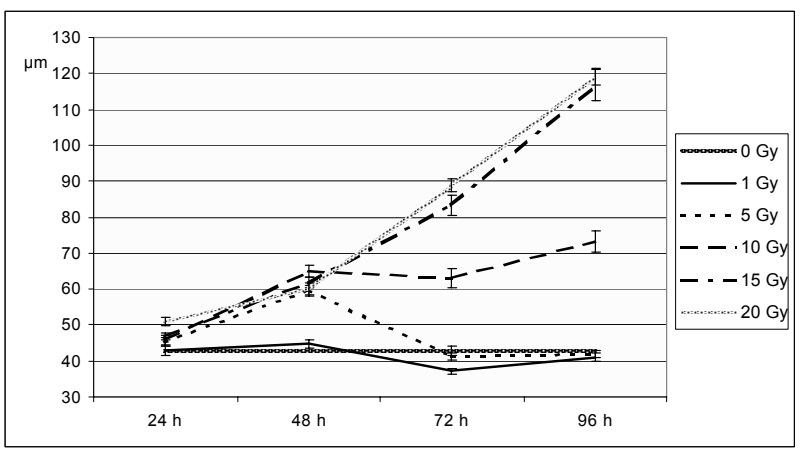

Fig. 6. Length of basal lamina of 10 enterocytes.

decrease of enterocyte height was observed.

\section{Length of basal lamina of enterocytes in jejunum (Fig. 6)}

In comparison with non-irradiated animals, the values of basal lamina were significantly higher in rats examined $48 \mathrm{~h}$ after irradiation with the dose of $5 \mathrm{~Gy}$, and at all time intervals in all rats irradiated with the dose of 10 Gy and higher. Maximum increase was measured at the time interval of $96 \mathrm{~h}$ after irradiation with the dose of $20 \mathrm{~Gy}$. The value measured at the time interval of $72 \mathrm{~h}$ after irradiation with the dose of 1 Gy was significantly lower.

\section{Enterocyte width in jejunum (Fig. 7)}

The changes of the enterocyte width in rat jejunum are very similar to those of the previous parameter. In comparison with non-irradiated animals, the values of the enterocyte width were significantly higher in rats examined $48 \mathrm{~h}$ after irradiation with the dose of 5 Gy, 48-96 h after irradiation with the doses of 10 and 15 Gy and at all time intervals after irradiation with the dose of 20 Gy. A maximum increase was measured at the time interval of $96 \mathrm{~h}$ after irradiation with the dose of 20 Gy. The value measured at the time interval of 24 and $72 \mathrm{~h}$ after irradiation with the dose of 1 Gy was significantly lower. 


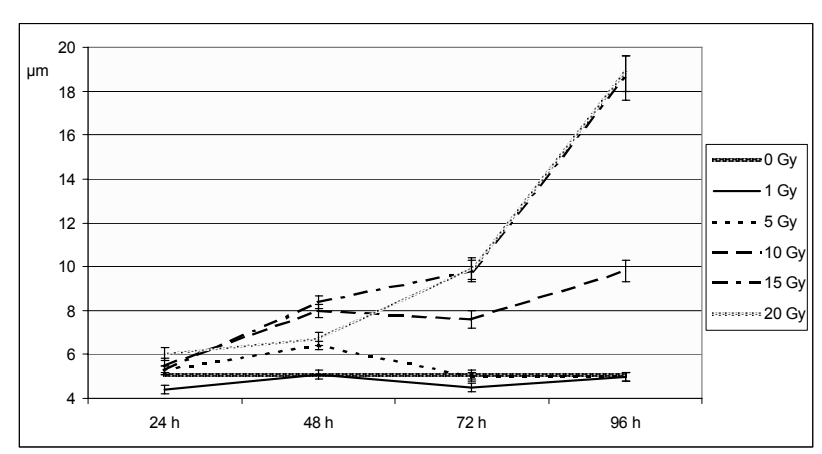

Fig. 7. Width of one enterocyte.

\section{Discussion}

Fajardo (1982) described a flattening of villus in developed radiation-induced intestine injury. In our animal experiments, the irradiation with sublethal doses did not influence the intercryptal distance in rat jejunum. After irradiation with lethal doses, the intercryptal distance increased in most groups proportionally with the dose. Besides the influence of reparation mechanisms, there is an enormous physiological variability in the morphology of villus, therefore the intercryptal distance cannot be considered as a suitable biodosimetric parameter.

According to Fajardo (1982), the main microscopic sign of radiation-induced intestine injury is flattening of enterocytes on the surface of villi. In our laboratory trial, we recognized that irradiation with sublethal (subthreshold) doses did not significantly change the height of the enterocyte both on the top and on the base of the villus. The early decrease of height of the enterocyte on the top of the villus irradiated with the dose of 5 Gy was completely compensated in 3 days. On the contrary, the early reduction in height of the enterocyte on the base of the villus irradiated with the dose of 5 Gy was completely compensated in 2 days. Even after irradiation with a supralethal dose of $10 \mathrm{~Gy}$, the height of the enterocyte on the base of the villus was temporarily compensated in 2 days, then significantly decreased.

After irradiation with supralethal doses, the height of the enterocytes on the top of the villus was significantly decreased. The height of the enterocytes on the base of the villus also significantly decreased, with the exception of temporarily compensation $48 \mathrm{~h}$ after irradiation with the dose of $10 \mathrm{~Gy}$. We suppose that the basal enterocytes attain higher and more rapid compensation (repopulation) than the enterocytes on the top of the villus.
The height of the enterocyte is dependent on the dose of irradiation and might be considered as a very sensitive and suitable biodosimetric marker after irradiation with the dose of $10 \mathrm{~Gy}$ and higher. Besides the number of crypts on the transverse section of jejunum, which is supposed as a fundamental biodosimetric marker (Österreicher et al. 2005, Potten 2004), the height of enterocyte might be another useful parameter under experimental conditions.

The flattening of enterocytes in intestinal radiation-induced pathology is a compensatory mechanism enabling lower amount of cells to cover a larger surface of villus (Fajardo 1982). According to our results, irradiation with sublethal doses does not influence the length of basal lamina of 10 enterocytes. Forty-eight hours after irradiation with the dose of 5 Gy the length became significantly increased, but by the third day the changes were compensated by repopulation of cells. After irradiation with supralethal doses, the length of basal lamina of 10 enterocytes significantly and dosedependently increased. Tendency towards proliferation in the crypts retards the development of intestinal damage, while the reparative mechanism of repopulation is insufficient later and after the higher doses.

Similarly, the width of one enterocyte increased after irradiation with supralethal doses. In comparison with the width of one enterocyte, the length of basal lamina for 10 enterocytes partially equilibrates the intercellular and intracellular variability and is more accurate. Moreover, the length of basal lamina of 10 enterocytes in all measurements was shorter than tenfold of one enterocyte width. We suppose that it might be a good marker of radiation-induced intestine injury under experimental conditions.

Experimental study is a predecessor of any clinical assessment of a specific marker. In clinical practice and, especially, under military field condition, the suitable biodosimetric parameter would serve as a certain guidance for evaluation of the absorbed dose in irradiated troops as well as in rescue workers. This is in accordance with tasks and Standardization Agreement of the North Atlantic Treaty Organization (Stanag 2002). Based on our unpublished experiments, biodosimetric markers detectable in proliferating cells cannot be used in non-proliferating cells (e.g. native lymphocytes). For clinical practice, the buccal or anal epithelium seems to be more accessible proliferating cell populations in the gastrointestinal tract that might reveal the gravity of radiation-induced damage. 


\section{Conclusion}

In our animal experiments, major morphological changes in small intestine mucosa developed rapidly and progressively during the first four days after irradiation with supralethal doses. The threshold dose for appearance and development of radiation-induced enteropathy in rats falls between 5 and $10 \mathrm{~Gy}$. Thus the threshold dose in rats seems to be very similar to that in humans and the course of the disease also resembles the clinical manifestation in patients. The threshold dose for radiation-induced enteritis is lower than $10 \mathrm{~Gy}$, which agrees with data listed in documents of the North Atlantic Treaty Organization (Stanag 2002).

In the laboratory trial, we were the first to quantify morphological post-irradiation changes in jejunum. Fajardo (1982) described these morphological changes, but he did not quantify them. With the exception of intercryptal distance, they might be considered as suitable biodosimetric markers under experimental conditions.

\section{Conflict of Interest}

There is no conflict of interest.

\section{References}

BRENNAN, PC, CARR KE, SEED T, MCCULLOUGH JS: Acute and protracted radiation effects on small intestinal morphological parameters. Int J Radiat Biol 73: 691-698, 1998.

BUELL MG, HARDING RK: Proinflammatory effects of local abdominal irradiation on rat gastrointestinal tract. Dig Dis Sci 34: 390-399, 1989.

CLASSEN J, BELKA C, PAULSEN F, BUDACH W, HOFFMANN W, BAMBERG M: Radiation-induced gastrointestinal toxicity. Strahlenther Onkol 174 (Suppl III): 82-84, 1998.

FAJARDO LF: Pathology of Radiation Injury. Masson Publishing, New York, 1982.

MACNAUGHTON WK: New insights into the pathogenesis of radiation-induced intestinal dysfunction. Aliment Pharmacol Ther 14: 523-528, 2000.

ÖSTERREICHER J, DRIÁK D, PRŮCHOVÁ Š, VILASOVÁ Z: Radiation enteritis: time- and dose-dependent reduction of jejunal crypts in the model of irradiated rats (in Czech). Military Medical Sheets 74: 77-80, 2005.

POTTEN CS: Radiation, the ideal cytotoxic agent for studying the cell biology of tissues such as the small intestine. Radiat Res 161: 123-136, 2004.

REGAUD C, NOGIER T, LACASSAGNE A: Sur les effets redoutables des irradiations étendues de l'abdomen et sur les lésions du tube digestif déterminées par les rayons de Röntgen. Arch D'Électr Méd Exp Clin 21: 321-334, 1912.

STANAG 2374: NATO Standardization Agreement No. 2374 (Allied Medical Publication No. 6), NATO Standardization Agency, 2002, pp 120.

WALSH D: Deep tissue traumatism from roentgen ray exposure. Br Med J 2: 272-273, 1897. 